\title{
Clinical complications during veno-arterial extracorporeal membrane oxigenation in post-cardiotomy and non post-cardiotomy shock: still the achille's heel
}

\author{
Valeria Lo Coco ${ }^{1,2 \#}$, Roberto Lorusso ${ }^{1}$, Giuseppe M. Raffa, ${ }^{1,2}$, Pietro Giorgio Malvindi ${ }^{3}$, Michele Pilato ${ }^{2}$, \\ Gennaro Martucci ${ }^{4}$, Antonio Arcadipane ${ }^{4}$, Kamil Zieliński ${ }^{5}$, Piotr Suwalski ${ }^{6,7}$, Mariusz Kowalewski ${ }^{6,8}$ \\ ${ }^{1}$ Department of Cardio-Thoracic Surgery, Heart and Vascular Centre, Maastricht University Medical Centre, Maastricht, The Netherlands; \\ ${ }^{2}$ Department of the Treatment and Study of Cardiothoracic Diseases and Cardiothoracic Transplantation, IRCCS-ISMETT (Istituto Mediterraneo \\ per i Trapianti e Terapie ad Alta Specializzazione), Palermo, Italy; ${ }^{3}$ GVM Care \& Research, Cardiac Surgery Unit, Santa Maria Hospital, Bari, \\ Italy; ${ }^{4}$ Department of Anaesthesia and Intensive Care, IRCCS-ISMETT, Palermo, Italy; ${ }^{5}$ Warsaw Medical University, Warsaw, Poland; ${ }^{6} \mathrm{Clinical}$ \\ Department of Cardiac Surgery, Central Clinical Hospital of the Ministry of Interior in Warsaw, Poland; ${ }^{7}$ Clinical Department of Cardiac Surgery, \\ Centre of Postgraduate Medical Education, Warsaw, Poland; ${ }^{8}$ Cardiothoracic Research Centre, Innovative Medical Forum, Bydgoszcz, Poland \\ Contributions: (I) Conception and design: V Lo Coco, GM Raffa, PG Malvindi, M Kowalewski; (II) Administrative support: None; (III) Provision of \\ study materials or patients: R Lorusso, GM Raffa, PG Malvindi, M Kowalewski; (IV) Collection and assembly of data: V Lo Coco, G Martucci, K \\ Zieliński; (V) Data analysis and interpretation: V Lo Coco, G Martucci, R Lorusso, M Pilato, A Arcadipane; (VI) Manuscript writing: All authors; (VII) \\ Final approval of manuscript: All authors. \\ \#These authors contributed equally to this work. \\ Correspondence to: Mariusz Kowalewski, MD. Department of Cardiac Surgery, Central Clinical Hospital of the Ministry of Interior and Administration \\ Warsaw, Wołoska 137 Str, 02-507 Warsaw, Poland. Email: kowalewskimariusz@gazeta.pl.
}

\begin{abstract}
Extracorporeal membrane oxygenation (ECMO) is life-saving for potentially reversible heart failure and respiratory injuries not responsive to conventional therapies. Technological innovations have produced over the years significant improvements in ECMO devices (pump, cannula design and oxygenator) and have allowed a better risk/benefit profile. Alongside with recognized advantages in the treatment of very sick patients, ECMO remains an invasive procedure for mechanical circulatory support (MCS) and it is associated with complications that strongly influence the prognosis. Current review was designed to provide a comprehensive outline on ECMO complications, analyzing risk factors and strategies of management, focusing on adult population undergoing veno-arterial ECMO (VA-ECMO) therapy.
\end{abstract}

Keywords: Extracorporeal life support (ECLS); extracorporeal membrane oxygenation (ECMO); mechanical circulatory support (MCS)

Submitted Nov 08, 2018. Accepted for publication Nov 19, 2018.

doi: $10.21037 /$ jtd.2018.11.103

View this article at: http://dx.doi.org/10.21037/jtd.2018.11.103

\section{Introduction}

Extracorporeal membrane oxygenation (ECMO) represents an established treatment for patients requiring temporary cardiopulmonary support (1-4) who have failed the maximal conventional medical management (5).

Thanks to the technological improvements that lead on the latest generation oxygenator, magnetic levitation pump and heparin-coated circuit, its use has exploded and progressed over the last decade with increasingly better outcomes (6-9).

Depending on the target, ECMO can be divided in two categories: veno-arterial (VA)-ECMO for cardiocirculatory and/or pulmonary support, and veno-venous (VV)-ECMO solely for pulmonary failure.

The indications for VA-ECMO are postcardiotomy cardiogenic shock (10-12), fulminant myocarditis $(13,14)$, 

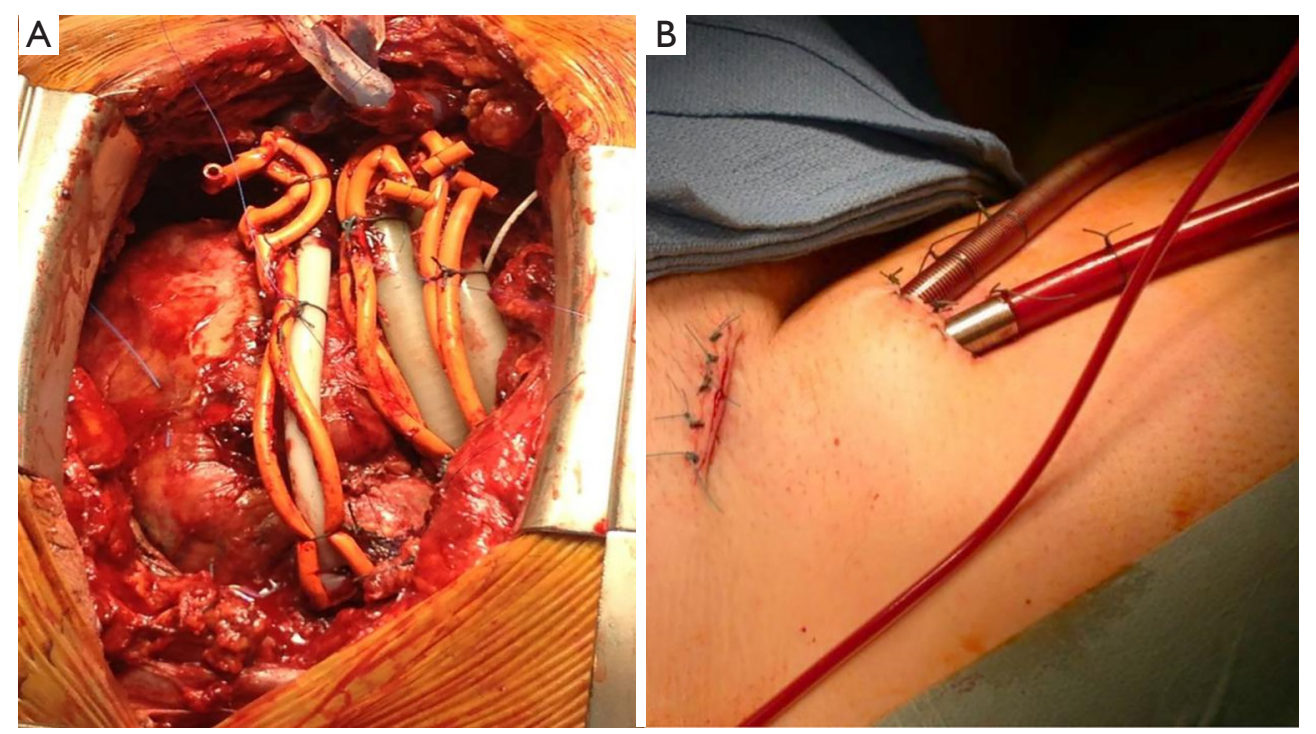

Figure 1 VA-ECMO. (A) Central VA-ECMO after sternotomy: cannulation of aorta and right atrium both fixed with two purse-string sutures and tourniquet (orange) venting the left ventricle through a cannula in the left atrium stabilized in the same way; (B) peripheral VAECMO: cannulation of femoral artery and vein after the surgical exposure of the vessels, the cannulas are under-exposed and the skin is closed. VA-ECMO, veno-arterial extracorporeal membrane oxygenation.

acute coronary syndrome (15), as a bridge to a longterm mechanical circulatory support (MCS) or heart transplantation (16), refractory cardiac arrest during cardiopulmonary resuscitation $(17,18)$ and primary graft failure (19). Further distinguishable are two configurations for the VA-ECMO: central and peripheral (Figure 1). Central cannulation for ECMO is the preferred modality in case of failed weaning from cardiopulmonary bypass (CPB) following cardiac surgery or in case of severe peripheral vasculopathy. Peripheral ECMO is established from peripheral vessels avoiding a thoracic surgical access; the possibility of rapid vessels cannulation provides undoubtable advantages, during cardiopulmonary resuscitation in the setting of refractory circulatory arrest (20-23). Extracorporeal Life Support Organization (ELSO) has been founded in 1989 by the need for a greater collaboration between specialists in the heart failure field. Every year, ELSO publishes the report with the outcomes of the ECMO patients treated with the member centers that are currently 310 . Of 9,025 adults with VA-ECMO for cardiac diseases from its inception in 1989 through July 1, 2016, 56\% have been weaned off ECMO and $41 \%$ discharged. There were 2,885 patients undergoing ECMO for cardiopulmonary resuscitation; in $39 \%$ of them it was possible the wean off from the assistance with a $10 \%$ in-hospital death rate. Cardiogenic shock was the most common cause with survival after admission of $42 \%$. The best prognosis has been associated with myocarditis with a $65 \%$ patients alive at discharge followed by cardiomyopathies with $51 \%$ (24) (Tables 1,2). Despite the increase in the volume of patients, the increased number of member centers and the technology improvements, the incidence of complications has remained largely unchanged (24-27). The most common complication has universally been bleeding (cannula and surgical site) from a minimum of $28 \%$ in 1997 report to $46.4 \%$ in the 2012 one. In the latest report, the oxygenator failure has decreased considerably $(0.8 \%)$ compared to the previous ones where it accounted for approximately $8-10 \%$. The percentage incidence of neurological complications and infection has been stable in the years' analyses. From these results, it's important to underline how a successful extracorporeal life support (ECLS) requires appropriate patient selection, skilled ECMO management with trained providers, and health care infrastructure that can help prevent or manage adverse events and hence requires considerable resources including finance and manpower (Table 3). 
Table 1 ECLS cases and survival to discharge

\begin{tabular}{lccc}
\hline Adult & Cases number & Survived ECLS, $n$ [\%] & Discharged, $\mathrm{n}[\%]$ \\
\hline Cardiac & 9,025 & $5,082[56]$ & $3,721[41]$ \\
ECPR & 2,885 & $1,137[39]$ & $848[29]$ \\
\hline
\end{tabular}

ECLS, extracorporeal life support; ECPR, ECLS to support cardiopulmonary resuscitation.

Table 2 ECLS for cardiac indications

\begin{tabular}{lcc}
\hline Diagnosis & Number & Survival, $\mathrm{n}[\%]$ \\
\hline Shock $^{*}$ & 2,083 & $882[42]$ \\
Cardiomyopathy & 704 & $358[51]$ \\
Myocarditis & 227 & $143[65]$ \\
Congenital defect & 420 & $156[37]$
\end{tabular}

*, cardiogenic shock.

\section{Pre-clinical ECMO studies and complications}

Animal models can help us to understand better the pathophysiology of the complications on ECMO and thus reduce their incidences.

$\mathrm{Mu}$ et al. showed in a piglet model how continuous renal replacement therapy, could reduce the inflammatory cytokine levels during the initial phase of ECMO following hemorrhage-reperfusion while maintaining cardiac output and oxygen utilization (28).

Another study proved in an ovine model that the ECMO therapy increases collagen-induced platelet aggregation, decreases factor VIII and von Willebrand factor; then it defines a decrease of fibrinogen levels and an alteration function in the first 24 hours (29).

Janák et al. demonstrated in a porcine model that the high VA-ECMO flow rates can influence a higher formation of microemboli in the blood circulation (30).

It's possible that different configuration for ECMO and then the different positioning of the cannulas (peripheral or central ECMO) may influence the brain differently. Gu et al. used a computation fluid dynamics (CFD) simulation which measures at the level of the carotid arteries the velocity of pulsatile flow from the left ventricle and non-pulsatile flow from a cannula in the ascending aorta. He reported a higher flow rate and a lower wall shear stress in the carotid artery with pECMO than with cECMO due to greater flow in the direction of the carotid artery (31). Kaufmann et al. used the CFD and particle image velocimetry in a silicone model and with both methods, there was a decrease in flow velocity in the carotid artery and an increase flow velocity in the descending aorta upon insertion of the cannula. This phenomenon was explained for the deviation of the cannula jet direction toward the descending aorta (32).

Ferreira $e t a l$. studied patients with Glasgow Coma Scale 3-8 and traumatic brain injury; they showed significantly higher IL-6 levels one day after admission to the intensive care unit compared to the other patients without brain injury (33). Bembea et al. reported plasma glial fibrillary acidic protein (GFAP) levels in patients on ECMO at 6, 12, and 24 hours after cannula insertion, and then every day until the end of ECMO significantly higher in patients with new-onset brain damage (34).

The finding of a prognostic clinical significance of increased markers of brain injury already at 24 hours of circulatory support should lead to future studies on biomarkers aiming a better definition of the impact of hemodynamic patterns based on different ECMO configurations. The utility of the animal models and the point-of-care tests are important for guiding the management during ECMO striking up the band for further studies and the training of a multidisciplinary team of expertise.

\section{Complications during VA-ECMO support}

Due to the critical state of patients suited for its use and the extracorporeal circuit itself, ECMO carries several possible complications that can have a relevant impact on the immediate and remote outcomes. A correct prevention and 
Table 3 Trend of complications for cardiac support in adults

\begin{tabular}{|c|c|c|c|c|}
\hline Complications & Year $1997(25)$ & Year 2004 (26) & Year $2012(27)$ & Year $2016(24)$ \\
\hline Oxygenator failure & 7 [29] & $16.4[27]$ & $15.1[36]$ & 6.6 \\
\hline Tubing rupture & $2[33]$ & $0.9[20]$ & $0.2[0]$ & - \\
\hline Cannula problems & $6[31]$ & $6.8[32]$ & $4.4[29]$ & - \\
\hline Surgical site hemorrhage & 28 [38] & $31.9[27]$ & $25.5[34]$ & 20.2 \\
\hline Cardiac tamponade & - & - & $5.7[27]$ & \\
\hline Pulmonary hemorrhage & - & - & - & 3.1 \\
\hline Gl hemorrhage & $2[11]$ & $2.4[15]$ & - & - \\
\hline CSN infarction & - & - & - & 3.8 \\
\hline $\mathrm{ICH}$ & - & - & $1.7[7]$ & - \\
\hline Clinical seizures & $11[28]$ & $4.8[12]$ & $2.1[15]$ & - \\
\hline Brain death & $6[0]$ & $7.9[0]$ & - & - \\
\hline Renal failure & - & - & - & 12.3 \\
\hline Hyperbilirubinemia & - & - & - & 12.2 \\
\hline Hemolisis & 6 [29] & 8.1 [34] & - & - \\
\hline Infections & - & - & - & 13 \\
\hline
\end{tabular}

Values are \% reported: \% is occurrence and in bracket the [\%] of survival accorded with the complications. *, it's included in the surgical site bleeding. Renal failure: serum creatinine $>3.0 \mathrm{mg} / \mathrm{dL}$; hyperbilirubinemia: total bilirubin $>2 \mathrm{mg} / \mathrm{dL}$ or indirect bilirubin $>15 \mathrm{mg} / \mathrm{dL}$. CSN, central nervous system; $\mathrm{ICH}$, intracranial hemorrhage.

an early recognition of specific signs can help to reduce the incidence of adverse events $(23,35,36)$ (Figure 2).

\section{Bleeding}

Patients while on ECMO can demonstrate various degrees of disseminated intravascular coagulation (DIC) and invariably and acquired von Willebrand disease due to the contact with the extracorporeal surface that activates the coagulation and therefore causes a consumption coagulopathy (37). Another cause is the sepsis in particular for patients in long time ECMO who are more susceptible to nosocomial infections often caused by multi-resistant bacteria (38). Bleeding is the most common complication especially in the postcardiotomy patients, ranging between $10-30 \%(24,39)$. The most frequent source of bleeding is the cannulation and surgical sites. Topical agents such as oxidized cellulose (Surgicel), gelatin sponge (Gelfoam), thrombin and fibrinogen sealant (Tissel) can be used at the surgical sites to help control oozing. It may occur also at airway and GI. In fact, routine procedures such as suctioning and bronchoscopy or urinary catheter insertion can trigger bleeding. The percentage of rethoracotomy and tamponade for hemorrhagic complications is around $40 \%$ (40). Bleeding depends on systemic anticoagulation, platelet dysfunction for contact and shear stress associated activation, the massive activation of coagulation and fibrinolysis during and after CEC, consumption coagulopathy and systemic inflammation. The use of heparinized circuits, the last generation oxygenators and the experience of high volume centers has prompted reports of low-dose heparin protocols for anticoagulation during ECMO, thus greatly decreasing hemorrhagic complications also in case of ECMO adopted in the perioperative 


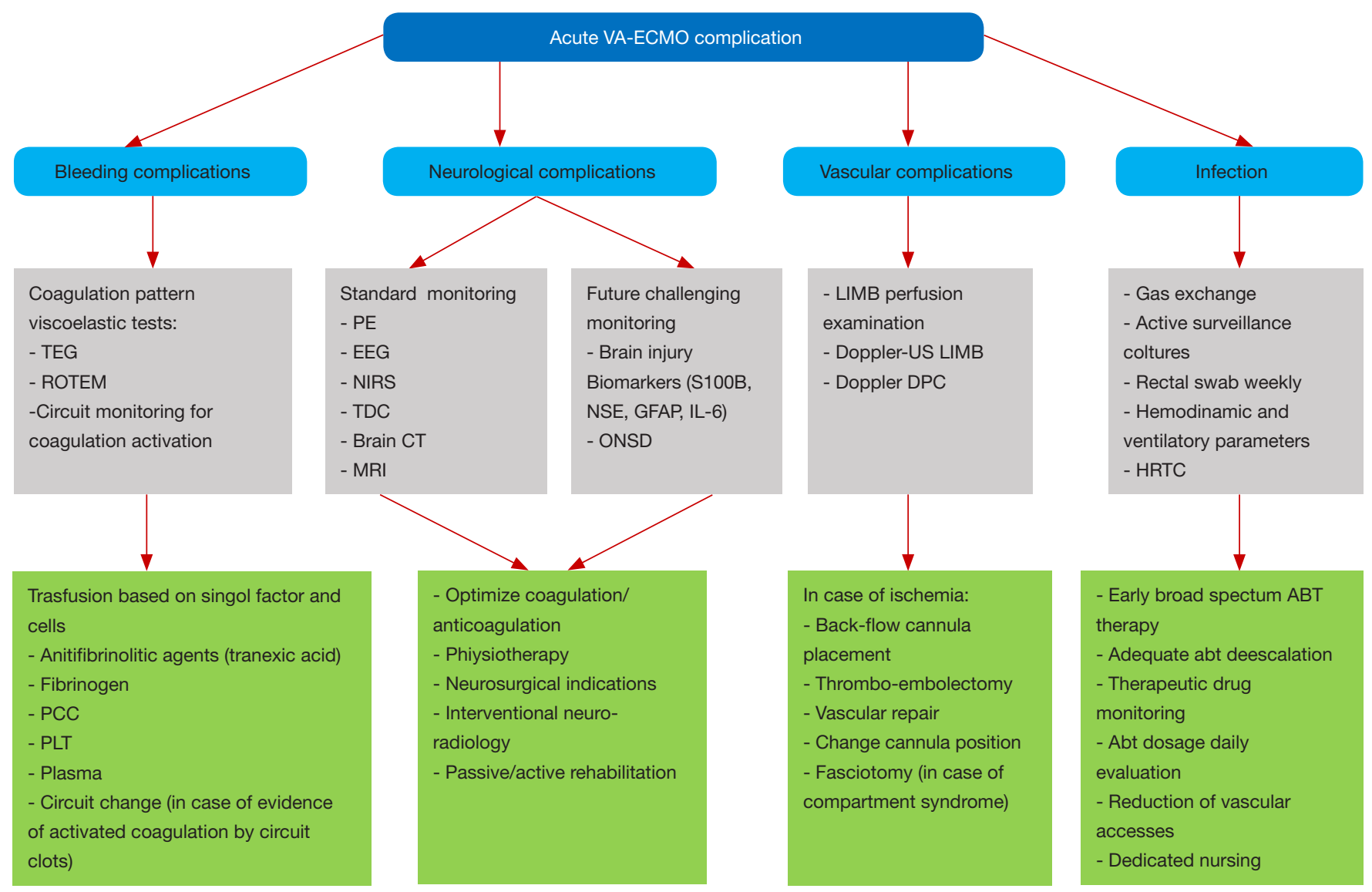

Figure 2 Flow chart of the assessment (grey) and management (green) of the ECMO complications. ABT, antibiotic therapy; DPC, distal perfusion catheter; EEG, electroencephalogram; GFAP, glial fibrillary acidic protein; HRTC, high-resolution chest tomography; NIRS, cerebral near infrared spectroscopy; PCC, prothrombin complex concentrate; PE, physical examination; PLT, platelet; ONSD, optic nerve sheath diameter; ROTEM, Rotational Thromboelastometry; TDC, transcranial Doppler; TE, thromboembolic; TEG, thromboelastography.

period $(41,42)$.

The replacement of blood products is not currently based on clear evidence and do not rely on accepted protocols in most centers but usually based on clinical experience, historical literature, and guideline recommendations than as a result of well conducted research studies. It is important to monitor the coagulation status [activated clotting time (ACT), activated partial thromboplastin time (aPTT), prothrombin time (PT), platelet count] especially before invasive procedure. The point of care viscoelastic tests [thromboelastography (TEG), rotational thromboelastometry (ROTEM)] allow a reduction of transfusions basing the treatment of hemorrhage on the single factors and single cells to be transfused, platelets and/or blood cells. This reduces the risks of fluid overload, immunosuppression, immunological and inflammatory activation due to allogeneic blood. Transfusions of packed red blood cells are given as needed to replace any blood loss and maintain a near normal hematocrit $(>35 \%)$. The target ACT is 210-230 seconds but once bleeding occurs, it may become 170-190 seconds reducing or holding the anticoagulation. The INR (PT) is a good test of the status of the hemostatic pathway. Fresh frozen plasma (FFP) is administered in aliquots of $5-10 \mathrm{~mL} / \mathrm{kg}$ as needed if the INR is $>1.5-2.0$ or in case altered viscoelastic tests if there is a significant bleeding. There is a significant relationship between platelet count and hemorrhagic complications on ECLS and platelet administration decreases the incidence of bleeding complications. It's important to maintain the platelet count greater than $50,000 / \mathrm{mm}^{3}$, transfusing 
$10 \mathrm{mg} / \mathrm{kg}$ of platelet or 1 unit per $5 \mathrm{~kg}$. Plasminogen inhibitors can be infused or heparin can be stopped for several hours even if they can increase the risk of thrombotic problem. Infusion of activated factor VII (rVIIa), usually given in a dose range of $40-90 \mu \mathrm{g} / \mathrm{kg}$, should only be considered when other options have failed and in case of refractory bleeding. In multiple series, following this treatment, patients had significant reduction in chest tube drainage and reduced need for packed red blood cell transfusions. The rate of thromboembolic complications $(25-30 \%)$ seen in the series reviewed, was not significantly higher than the rate of the same complications in ECLS patients that did not receive rVIIa. However, its use must be extremely careful and indeed some centers administer lower doses of rVIIa $(25-50 \mu \mathrm{g} / \mathrm{kg})$; if more than one dose is required, they do not repeat the dose more than every two to four hours. The best possible prevention of bleeding/thrombotic events appears to be disturbing as little as possible the natural balance between coagulation and anticoagulation. This translates in daily practice into the administration of low doses of heparin and attention to supplementation of antithrombin III (42-44).

\section{Thromboembolic complications}

Thromboembolic complications have decreased due to the use of biocompatible materials. Because of blood-surface interaction, clots can form in the circuit and originate to embolic events. Thrombi can appear at any point in the circuit, rare in the literature are the thrombosis of the pump, the most common are the micro thrombosis of the oxygenator (45). A massive gas embolism can occur for formation of air bubbles entrained in the negativepressure portion of the circuit. Fundamental is a correct balance between hemostasis and thrombosis to avoid neurological, bleeding and thromboembolic complications. Sometimes this balance is more difficult to achieve due to the development of a coagulopathy. Thrombocytopenia is common in ECLS patients and it may be a consequence of the primary disease, drug induced, or caused by blood exposure to the circuit surface. Even if the platelet count is over the minimum level, the platelet function can be impaired (46). A rare condition associated with heparin anticoagulation is heparin-induced thrombotic thrombocytopenia (HITT) which is characterized by multiple white arterial thrombi and platelet count less than 10,000 . It's reasonable to use a different anti-coagulant such as Argatroban because its half life is short and a similar ACT target range is effective. When the pump suction exceeds the blood drainage (high inlet pressures) for a high flow rate through a very small orifice or for a high level of occlusion in the post-pump circuit, hemolysis may occur with elevated plasma free hemoglobin and hyperbilirubinemia.

\section{Neurological complications}

Major complications consist of central nervous system hemorrhage (2\%) and infarction (4\%). Other very subtle neurological complications, which must therefore be investigated in a specific way are generalized epileptic status and other neurological complications related to the underlying disease that had led to the ECMO (47-50). The most devastating is the intracranial hemorrhage (ICH) which is associated with a poor prognosis, usually fatal (51-54). Pre-ECMO factors can influence its incidence. Cardiac arrest before the mechanical support may be linked with ICH for the development of anoxic brain injury or brain infarction with subsequent hemorrhagic transformation $(48,49,55)$. Sepsis and influenza are associated in many studies (52). It is thought to be associated with alterations of the blood-brain barrier that could predispose to brain injury $(56,57)$. Renal failure and renal replacement therapy cause platelet dysfunction and increase their consumption, facilitating the ICH $(55,58)$. The worse outcome in ICH is associated with hemolysis and thrombocytopenia $(48,49)$. Other risk factors are younger age, female gender and lower body surface area (59). In addition to hemorrhage, infarction (1-8\%) and seizures with cerebral edema $(2-10 \%)$ are frequent complications. Thromboembolic events can lead to stroke in different parts of the brain and are often related to microembolism of blood clot or air (60). Pre-ECMO lactic acid greater than $10 \mathrm{mmol} / \mathrm{L}$ could be an independent predictor of ischemic stroke occurring during ECMO support as a marker of tissue hypoperfusion (61). Moreover, during VAECMO cerebral perfusion is mainly non-pulsatile (unless combined with an intra-aortic balloon pump), although it's unclear if it's correlated to neurological complication. Neurological diagnosis is insidious because of deep sedation and the low efficacy of neurological exams consequently [computed tomography (CT) scan, magnetic resonance imaging (MRI)]. In addition to a neurological monitoring [protein biomarkers of brain injury, cerebral near infrared spectroscopy (NIRS), and the transcranial Doppler (TCD)], there are other methods to estimate the brain injury such as the seizure activity, abnormal electroencephalograms, 
and somatosensory evoked potentials. In case of intracranial hypertension, a recent increasingly monitor tool adopted is the optic nerve sheath diameter (ONSD) that seems to be able to detect early, and in a safe and non-invasive way the intracranial hypertension, helping thus in early detection of severe intracranial conditions (62). The brain biomarkers are: $\mathrm{S} 100 \beta$ which elevated levels have been associated with ICH in infants treated with ECMO, and high GFAP during ECMO has been associated with acute brain injury and death the neuron-specific enolase (NSE) and the IL-6 which is a pro-inflammatory cytokine. These markers are secreted by brain tissue into the systemic circulation during brain damage and can increase in concentration following hemodynamic damage to the blood-brain barrier $(63,64)$.

\section{Infections}

Infections, mainly nosocomial infections, are common complications in ECMO patients due to several predisposing factors such as comorbidities, immunocompromised status associated to transfusions and critical illness, and invasiveness of ECMO and other life-support sustain like multiple vascular catheters, invasive mechanical ventilation and continuous renal replacement therapy (65). Septic complications (13\%) are often associated with long permanence of exogenous material in direct contact with the heart and the vessels. They increase especially in case of sternotomy, long-term support and ventilatory support, longer length of stay in the ICUs and in the hospital. They are mainly dependent on the respiratory and urinary system and often are associated with sepsis. Less common albeit equally important are surgical site infections (mainly the groin) with a localization in the cannula $(66,67)$. The clinical diagnosis of infection in ECMO patients is particularly challenging since the clinical picture is often vague (68). Fever is frequently non-apparent since body temperature is controlled by the heat exchanger and signs of systemic activation of inflammation are invariably present, partially triggered by ECMO circuit itself. It's important to monitor the white blood cell (WBC) and the inflammation indices [C-reactive protein (CRP), procalcitonin (PCT)] that are altered in presence of an extracorporeal circuit but may be a relevant alarm sign for early institution of empiric antibiotic therapy. A broad-spectrum empiric antimicrobial therapy should be instituted early and prompt de-escalation when the results of microbiological cultures become known should be afforded $(69,70)$. Special concern should be given to the drug dosing during ECMO support and consequently also for antibiotics, antivirals and antifungal agents. Therefore, blood volume in the ECMO increase the volume of distribution and moreover ECMO circuit and membrane result in the sequestration of drugs altering the pharmacokinetic of several antibiotics preventing in this way the deployment of an adequate concentration in the inoculums site. Giving these premises, regular therapeutic drug monitoring daily reevaluation of drug dose should be a standard of care during ECMO (71).

\section{Vascular complications}

Another major complications on ECMO are vascular. It can be derived from a difficult cannulation due to anatomical reasons (size, stenosis, calcifications, anatomical variations, previous surgeries and obesity) and clinical condition of the patient (absence of pulsatility during low-flow states or cardiac arrest, vasoconstriction) (72). Arterial cannulation may lead to serious complications particularly with a percutaneous technique regardless of the use of echo guided strategy (73-75). Perforation of the posterior wall of the vessel is associated with bleeding and inadequate perfusion. Compartment syndrome or retroperitoneal hematoma, may be devastating entity; depending on the site of vascular injury. Other complications are the formation of arteriovenous fistula or pseudoaneurysm which need a surgical repair $(<5 \%)$. Guidewires and dilatators can also cause arterial dissection leading to extravascular cannula positioning and inability to circulate blood (76,77). The femoral arterial cannulation, the large bore cannulas and the hemodynamic instability during peripheral VA-ECMO places the limb at risk of ischemia due to thromboembolic complications. Larger cannulas (>20 Fr), female gender, younger patients and the presence of peripheral disease increase the risk. The common femoral artery is smaller in women and its size increases with age (78). Difficult bedside cannulation and the presence of peripheral vascular disease compromises the blood flow of the lower extremity resulting in an additional risk factor. The insertion of a distal perfusion cannula which consists in a 4 or $5 \mathrm{Fr}$ anterograde catheter placed in the proximal femoral artery and connected via a T-connector, to the side port of the arterial cannula, should be always considered to provide adequate limb perfusion $(79,80)$ (Figure 3).

\section{VA-ECMO specific complications}

VA-ECMO is associated with specific complications such 

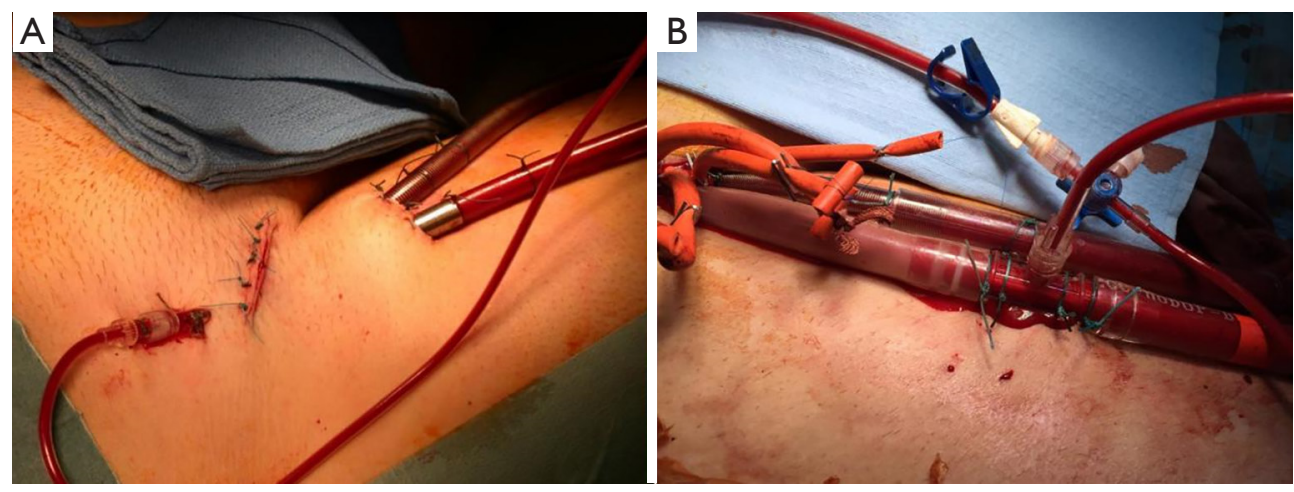

Figure 3 Distal perfusion catheter. (A) Selective perfusion of superficial femoral artery and (B) T-connector in the arterial inflow cannula.

as the cardiac thrombosis and the coronary or cerebral hypoxia. There is retrograde blood flow in the ascending aorta whenever the femoral artery and vein are used for the MCS. Stasis of the blood can occur if left ventricular output is not maintained, which may result in thrombosis. $(81,82)$. In fact, during VA-ECMO, fully saturated blood infused into the femoral artery from the ECMO circuit will perfuse preferentially the lower extremities and the abdominal viscera. Blood ejected from the heart will perfuse selectively the heart, brain and upper extremities. As a result, the oxyhemoglobin saturation of the blood perfusing the lower extremities and abdominal viscera may be substantially higher than that perfusing the heart, brain and upper extremities. Differential hypoxia also is known as north-south syndrome, proximal-distal syndrome, and Harlequin syndrome. To avoid this complication, arterial oxyhemoglobin saturation should be monitored in the right upper extremity. Poor arterial oxyhemoglobin saturation measured from the upper extremity is corrected by infusing some oxygenated blood into the right atrium (called VA-V access) $(83,84)$. A particular problem associated with VAECMO is the LV distention. An evidence of pulmonary edema on the chest radiograph or edema fluid frothing up the endotracheal tube can be the first manifestation of this problem. A transesophageal echocardiography can confirm the diagnosis identifying a severely dilated LV. The presence of mitral or aortic regurgitation can exacerbate the problem, and an increase in pump flow helps to reduce the pulmonary flow, ameliorating the condition. LV distention is not a problem exclusively related to central VA-ECMO; in patient with peripheral VA-ECMO, despite adequate left ventricular unloading, there is still returning blood flow to the left atrium, principally due to the bronchial circulation. Consequently, if LV contractility is profoundly reduced, one can anticipate an increase in left heart pressures resulting in LV distention. The increase in wall stress associated with LV distention not only increases myocardial energy consumption resulting in ischemia but also reduces the likelihood of ventricular recovery. A surgical or percutaneous $L V$ vent insertion must be performed in these cases (85-87).

\section{Conclusions}

ECMO represents the first life-saving system for advanced heart failure, among its advantages are the wide availability in most hospitals and the speed of implantation. Despite the improvements in the technology that led to the latest generation oxygenators, magnetic levitation pumps and heparin-coated circuits, ECMO is not free from risk and its indication should be subordinated to an interdisciplinary team. Complications are still associated with a poor prognosis, and influence morbidity and mortality. So, they play a major role in successful outcomes. Nowadays MCS and in particular the ECMO can't be excluded from the therapeutic range of patients with cardiogenic shock or cardiac arrest. Greater knowledge of the pathophysiology underlying complications, early detection and an accurate monitoring can help to reduce their incidence and improving the prognosis and outcome.

\section{Acknowledgements}

None.

\section{Footnote}

Conflicts of Interest: The authors have no conflicts of interest 
to declare.

Informed Consent: Written informed consent was obtained from the patient for publication of this manuscript and any accompanying images.

\section{References}

1. Schuerer DJ, Kolovos NS, Boyd KV, et al. Extracorporeal membrane oxygenation: current clinical practice, coding, and reimbursement. Chest 2008;134:179-84.

2. Lewandowski K. Extracorporeal membrane oxygenation for severe acute respiratory failure. Crit Care 2000;4:156-68.

3. Weber TR. Extending the uses of ECMO. Chest 2004;126:9-10.

4. Pillai AK, Bhatti Z, Bosserman AJ, et al. Management of vascular complications of extra-corporeal membrane oxygenation. Cardiovasc Diagn Ther 2018;8:372-7.

5. Gaffney AM, Wildhirt SM, Griffin MJ, et al. Extracorporeal life support. BMJ 2010;341:c5317.

6. Karagiannidis C, Brodie D, Strassmann S, et al. Extracorporeal membrane oxygenation: evolving epidemiology and mortality. Intensive Care Med 2016;42:889-96.

7. Sauer CM, Yuh DD, Bonde P. Extracorporeal membrane oxygenation use has increased by $433 \%$ in adults in the United States from 2006 to 2011. ASAIO J 2015;61:31-6.

8. Conrad SA, Broman LM. The Extracorporeal Life Support Organization Maastricht Treaty for Nomenclature in Extracorporeal Life Support. A Position Paper of the Extracorporeal Life Support Organization. Am J Respir Crit Care Med 2018;198:447-51.

9. Di Nardo M, Vercaemst L, Swol J. A narrative review of the technical standards for extracorporeal life support devices (pumps and oxygenators) in Europe. Perfusion 2018;33:553-61.

10. Elsharkawy HA, Li L, Esa WA, et al. Outcome in patients who require venoarterial extracorporeal membrane oxygenation support after cardiac surgery. J Cardiothorac Vasc Anesth 2010;24:946-51.

11. Rastan AJ, Dege A, Mohr M, et al. Early and late outcomes of 517 consecutive adult patients treated with extracorporeal membrane oxygenation for refractory postcardiotomy cardiogenic shock. J Thorac Cardiovasc Surg 2010;139:302-11, 311.e1.

12. Wu MY, Lin PJ, Lee MY, et al. Using extracorporeal life support to resuscitate adult postcardiotomy cardiogenic shock: treatment strategies and predictors of short-term and midterm survival. Resuscitation 2010;81:1111-6.

13. Hsu KH, Chi NH, Yu HY, et al. Extracorporeal membranous oxygenation support for acute fulminant myocarditis: analysis of a single center's experience. Eur J Cardiothorac Surg 2011;40:682-8.

14. Pozzi M, Banfi C, Grinberg D, et al. Veno-arterial extracorporeal membrane oxygenation for cardiogenic shock due to myocarditis in adult patients. J Thorac Dis 2016;8:E495-502.

15. Kim H, Lim SH, Hong J, et al. Efficacy of veno-arterial extracorporeal membrane oxygenation in acute myocardial infarction with cardiogenic shock. Resuscitation 2012;83:971-5.

16. Pagani FD, Aaronson KD, Swaniker F, et al. The use of extracorporeal life support in adult patients with primary cardiac failure as a bridge to implantable left ventricular assist device. Ann Thorac Surg 2001;71:S7781; discussion S82-5.

17. Chung SY, Sheu JJ, Lin YJ, et al. Outcome of patients with profound cardiogenic shock after cardiopulmonary resuscitation and prompt extracorporeal membrane oxygenation support. A single-center observational study. Circ J 2012;76:1385-92.

18. Wu MY, Lee MY, Lin CC, et al. Resuscitation of nonpostcardiotomy cardiogenic shock or cardiac arrest with extracorporeal life support: the role of bridging to intervention. Resuscitation 2012;83:976-81.

19. Loforte A, Murana G, Cefarelli M, et al. Role of IntraAortic Balloon Pump and Extracorporeal Membrane Oxygenation in Early Graft Failure After Cardiac Transplantation. Artif Organs 2016;40:E136-45.

20. Makdisi G, Wang IW. Extra Corporeal Membrane Oxygenation (ECMO) review of a lifesaving technology. J Thorac Dis 2015;7:E166-76.

21. Loforte A, Montalto A, Ranocchi F, et al. Peripheral extracorporeal membrane oxygenation system as salvage treatment of patients with refractory cardiogenic shock: preliminary outcome evaluation. Artif Organs 2012;36:E53-61.

22. Abrams D, Garan AR, Abdelbary A, et al. Position paper for the organization of ECMO programs for cardiac failure in adults. Intensive Care Med 2018;44:717-29.

23. Raffa GM, Kowalewski M, Brodie D, et al. Meta-Analysis of Peripheral or Central ECMO in Postcardiotomy and Non-Postcardiotomy Shock. Ann Thorac Surg 2018. [Epub ahead of print].

24. Thiagarajan RR, Barbaro RP, Rycus PT, et al. 
Extracorporeal Life Support Organization Registry International Report 2016. Asaio j 2017;63:60-7.

25. Conrad SA, Rycus PT. Extracorporeal life support 1997. ASAIO J 1998;44:848-52.

26. Conrad SA, Rycus PT, Dalton H, Extracorporeal Life Support Registry Report 2004, ASAIO J 2005;51:4-10.

27. Paden ML, Conrad SA, Rycus PT, et al. Extracorporeal Life Support Organization Registry Report 2012. Asaio j 2013;59:202-10.

28. Mu TS, Palmer EG, Batts SG, et al. Continuous renal replacement therapy to reduce inflammation in a piglet hemorrhage-reperfusion extracorporeal membrane oxygenation model. Pediatr Res 2012;72:249-55.

29. Passmore MR, Fung YL, Simonova G, et al. Evidence of altered haemostasis in an ovine model of venovenous extracorporeal membrane oxygenation support. Crit Care 2017;21:191.

30. Janák D, Hála P, Mlček M, et al. Detection of microembolic signals in the common carotid artery using Doppler sonography in the porcine model of acute heart failure treated by veno-arterial extracorporeal membrane oxygenation. Physiol Res 2017;66:S529-36.

31. Gu K, Zhang Y, Gao B, et al. Hemodynamic Differences Between Central ECMO and Peripheral ECMO: A Primary CFD Study. Med Sci Monit 2016;22:717-26.

32. Kaufmann TA, Neidlin M, Busen M, et al. Implementation of intrinsic lumped parameter modeling into computational fluid dynamics studies of cardiopulmonary bypass. J Biomech 2014;47:729-35.

33. Ferreira LC, Regner A, Miotto KD, et al. Increased levels of interleukin-6, -8 and -10 are associated with fatal outcome following severe traumatic brain injury. Brain Inj 2014;28:1311-6.

34. Bembea MM, Savage W, Strouse JJ, et al. Glial fibrillary acidic protein as a brain injury biomarker in children undergoing extracorporeal membrane oxygenation. Pediatr Crit Care Med 2011;12:572-9.

35. Brown KL, MacLaren G, Marino BS. Looking beyond survival rates: neurological outcomes after extracorporeal life support. Intensive Care Med 2013;39:1870-2.

36. Smedira NG, Moazami N, Golding CM, et al. Clinical experience with 202 adults receiving extracorporeal membrane oxygenation for cardiac failure: survival at five years. J Thorac Cardiovasc Surg 2001;122:92-102.

37. Oezpeker C, Zittermann A. Changes in Von Willebrand factor profile predicts clinical outcomes in patients on mechanical circulatory support. J Card Surg 2018;33:693-702.
38. Kim HS, Cheon DY, Ha SO, et al. Early changes in coagulation profiles and lactate levels in patients with septic shock undergoing extracorporeal membrane oxygenation. J Thorac Dis 2018;10:1418-30.

39. Bartlett RH, Gattinoni L. Current status of extracorporeal life support (ECMO) for cardiopulmonary failure. Minerva Anestesiol 2010;76:534-40.

40. Cheng R, Hachamovitch R, Kittleson M, et al. Complications of extracorporeal membrane oxygenation for treatment of cardiogenic shock and cardiac arrest: a meta-analysis of 1,866 adult patients. Ann Thorac Surg 2014;97:610-6.

41. Di Lorenzo G, Martucci G, Sciacca S, et al. Dysfunction of mechanical mitral prosthesis at $33 \mathrm{rd}$ week of pregnancy: ECMO support as a complex strategy for the mother and the fetus. Perfusion 2016;31:611-3.

42. Martucci G, Panarello G, Occhipinti G, et al. Anticoagulation and Transfusions Management in VenoVenous Extracorporeal Membrane Oxygenation for Acute Respiratory Distress Syndrome: Assessment of Factors Associated With Transfusion Requirements and Mortality. J Intensive Care Med 2017. [Epub ahead of print].

43. Wittenstein B, Ng C, Ravn H, et al. Recombinant factor VII for severe bleeding during extracorporeal membrane oxygenation following open heart surgery. Pediatr Crit Care Med 2005;6:473-6.

44. Anselmi A, Guinet P, Ruggieri VG, et al. Safety of recombinant factor VIIa in patients under extracorporeal membrane oxygenation. Eur J Cardiothorac Surg 2016;49:78-84.

45. Zanatta P, Forti A, Bosco E, et al. Microembolic signals and strategy to prevent gas embolism during extracorporeal membrane oxygenation. J Cardiothorac Surg 2010;5:5.

46. Cornell T, Wyrick P, Fleming G, et al. A case series describing the use of argatroban in patients on extracorporeal circulation. ASAIO J 2007;53:460-3.

47. Lorusso R, Taccone FS, Belliato M, et al. Brain monitoring in adult and pediatric ECMO patients: the importance of early and late assessments. Minerva Anestesiol 2017;83:1061-74.

48. Lorusso R, Gelsomino S, Parise O, et al. Neurologic Injury in Adults Supported With Veno-Venous Extracorporeal Membrane Oxygenation for Respiratory Failure: Findings From the Extracorporeal Life Support Organization Database. Crit Care Med 2017;45:1389-97.

49. Lorusso R, Barili F, Mauro MD, et al. In-Hospital Neurologic Complications in Adult Patients Undergoing Venoarterial Extracorporeal Membrane Oxygenation: 
Results From the Extracorporeal Life Support Organization Registry. Crit Care Med 2016;44:e964-72.

50. Lorusso R. Extracorporeal life support and neurologic complications: still a long way to go. J Thorac Dis 2017;9:E954-6.

51. Martucci G, Re VL, Marrone G, et al. Intracranial hemorrhage during extracorporeal membrane oxygenation: does family history play a role? Neurol Sci 2015;36:1523-5.

52. Martucci G, Lo Re V, Arcadipane A. Neurological injuries and extracorporeal membrane oxygenation: the challenge of the new ECMO era. Neurol Sci 2016;37:1133-6.

53. Cavayas YA, Del Sorbo L, Fan E. Intracranial hemorrhage in adults on ECMO. Perfusion 2018;33:42-50.

54. Fletcher-Sandersjöö A, Thelin EP, Bartek J Jr, et al. Incidence, Outcome, and Predictors of Intracranial Hemorrhage in Adult Patients on Extracorporeal Membrane Oxygenation: A Systematic and Narrative Review. Front Neurol 2018;9:548.

55. Fletcher Sandersjöö A, Bartek J Jr, Thelin EP, et al. Predictors of intracranial hemorrhage in adult patients on extracorporeal membrane oxygenation: an observational cohort study. J Intensive Care 2017;5:27.

56. Nasr DM, Rabinstein AA. Neurologic Complications of Extracorporeal Membrane Oxygenation. J Clin Neurol 2015;11:383-9.

57. Chow FC, Edlow BL, Frosch MP, et al. Outcome in patients with $\mathrm{H} 1 \mathrm{~N} 1$ influenza and cerebrovascular injury treated with extracorporeal membrane oxygenation. Neurocrit Care 2011;15:156-60.

58. Lockie CJA, Gillon SA, Barrett NA, et al. Severe Respiratory Failure, Extracorporeal Membrane Oxygenation, and Intracranial Hemorrhage. Crit Care Med 2017;45:1642-9.

59. Kasirajan V, Smedira NG, McCarthy JF, et al. Risk factors for intracranial hemorrhage in adults on extracorporeal membrane oxygenation. Eur J Cardiothorac Surg 1999;15:508-14.

60. Mehta A, Ibsen LM. Neurologic complications and neurodevelopmental outcome with extracorporeal life support. World J Crit Care Med 2013;2:40-7.

61. Omar HR, Mirsaeidi M, Shumac J, et al. Incidence and predictors of ischemic cerebrovascular stroke among patients on extracorporeal membrane oxygenation support. J Crit Care 2016;32:48-51.

62. Robba C, Santori G. Optic nerve sheath diameter measured sonographically as non-invasive estimator of intracranial pressure: a systematic review and meta- analysis. Intensive Care Med 2018;44:1284-94.

63. Robba C, Cardim D, Tajsic T, et al. Ultrasound noninvasive measurement of intracranial pressure in neurointensive care: A prospective observational study. PLoS Med 2017;14:e1002356.

64. Gazzolo D, Masetti P, Meli M, et al. Elevated S100B protein as an early indicator of intracranial haemorrhage in infants subjected to extracorporeal membrane oxygenation. Acta Paediatr 2002;91:218-21.

65. Schmidt M, Brechot N, Hariri S, et al. Nosocomial infections in adult cardiogenic shock patients supported by venoarterial extracorporeal membrane oxygenation. Clin Infect Dis 2012;55:1633-41.

66. Sun HY, Ko WJ, Tsai PR, et al. Infections occurring during extracorporeal membrane oxygenation use in adult patients. J Thorac Cardiovasc Surg 2010;140:1125-32.e2.

67. Hsu MS, Chiu KM, Huang Y'T, et al. Risk factors for nosocomial infection during extracorporeal membrane oxygenation. J Hosp Infect 2009;73:210-6.

68. Grasselli G, Scaravilli V, Di Bella S, et al. Nosocomial Infections During Extracorporeal Membrane Oxygenation: Incidence, Etiology, and Impact on Patients' Outcome. Crit Care Med 2017;45:1726-33.

69. Aubron C, Cheng AC, Pilcher D, et al. Infections acquired by adults who receive extracorporeal membrane oxygenation: risk factors and outcome. Infect Control Hosp Epidemiol 2013;34:24-30.

70. Tanaka D, Hirose H, Cavarocchi N, et al. The Impact of Vascular Complications on Survival of Patients on Venoarterial Extracorporeal Membrane Oxygenation. Ann Thorac Surg 2016;101:1729-34.

71. Hahn J, Choi JH, Chang MJ. Pharmacokinetic changes of antibiotic, antiviral, antituberculosis and antifungal agents during extracorporeal membrane oxygenation in critically ill adult patients. J Clin Pharm Ther 2017;42:661-71.

72. Barbone A, Malvindi PG, Sorabella RA, et al. 6 months of "temporary" support by Levitronix left ventricular assist device. Artif Organs 2012;36:639-42.

73. Aziz F, Brehm CE, El-Banyosy A, et al. Arterial complications in patients undergoing extracorporeal membrane oxygenation via femoral cannulation. Ann Vasc Surg 2014;28:178-83.

74. Roussel A, Al-Attar N, Alkhoder S, et al. Outcomes of percutaneous femoral cannulation for venoarterial extracorporeal membrane oxygenation support. Eur Heart J Acute Cardiovasc Care 2012;1:111-4.

75. Bisdas T, Beutel G, Warnecke G, et al. Vascular complications in patients undergoing femoral cannulation 
for extracorporeal membrane oxygenation support. Ann Thorac Surg 2011;92:626-31.

76. Zimpfer D, Heinisch B, Czerny M, et al. Late vascular complications after extracorporeal membrane oxygenation support. Ann Thorac Surg 2006;81:892-5.

77. Foley PJ, Morris RJ, Woo EY, et al. Limb ischemia during femoral cannulation for cardiopulmonary support. J Vasc Surg 2010;52:850-3.

78. Huang SC, Yu HY, Ko WJ, et al. Pressure criterion for placement of distal perfusion catheter to prevent limb ischemia during adult extracorporeal life support. J Thorac Cardiovasc Surg 2004;128:776-7.

79. Russo CF, Cannata A, Vitali E, et al. Prevention of limb ischemia and edema during peripheral venoarterial extracorporeal membrane oxygenation in adults. J Card Surg 2009;24:185-7.

80. Lamarche Y, Chow B, Bedard A, et al. Thromboembolic events in patients on extracorporeal membrane oxygenation without anticoagulation. Innovations (Phila) 2010;5:424-9.

81. Slottosch I, Liakopoulos O, Kuhn E, et al. Outcomes after peripheral extracorporeal membrane oxygenation therapy for postcardiotomy cardiogenic shock: a single-center

Cite this article as: Lo Coco V, Lorusso R, Raffa GM, Malvindi PG, Pilato M, Martucci G, Arcadipane A, Zieliński K, Suwalski P, Kowalewski M. Clinical complications during veno-arterial extracorporeal membrane oxigenation in postcardiotomy and non post-cardiotomy shock: still the achille's heel. J Thorac Dis 2018;10(12):6993-7004. doi: 10.21037/ jtd.2018.11.103 experience. J Surg Res 2013;181:e47-55.

82. Biscotti M, Bacchetta M. The "sport model": extracorporeal membrane oxygenation using the subclavian artery. Ann Thorac Surg 2014;98:1487-9.

83. Alexis-Ruiz A, Ghadimi K, Raiten J, et al. Hypoxia and Complications of Oxygenation in Extracorporeal Membrane Oxygenation. J Cardiothorac Vasc Anesth 2018. [Epub ahead of print].

84. Meani P, Gelsomino S, Natour E, et al. Modalities and Effects of Left Ventricle Unloading on Extracorporeal Life support: a Review of the Current Literature. Eur J Heart Fail 2017;19 Suppl 2:84-91.

85. Lorusso R. Are two crutches better than one? The ongoing dilemma on the effects and need for left ventricular unloading during veno-arterial extracorporeal membrane oxygenation. Eur J Heart Fail 2017;19:413-5.

86. Meani P, Pappalardo F. The step forward for VA-ECMO: left ventricular unloading! J Thorac Dis 2017;9:4149-51.

87. Camboni D, Schmid C. To vent or not on veno-arterial extracorporeal membrane oxygenation, does it improve myocardial recovery and outcome? J Thorac Dis 2017;9:4915-8. 